\title{
24. Freiwilliges Engagement von Frauen und Männern in Deutschland
}

\author{
Claudia Vogel, Julia Simonson, Jochen P. Ziegelmann \\ \& Clemens Tesch-Römer
}

\subsection{Einleitung}

In diesem Kapitel stehen die Befunde zum freiwilligen Engagement von Frauen und Männern im Mittelpunkt, die im vorliegenden Bericht zum freiwilligen Engagement in Deutschland in zahlreichen Kapiteln systematisch präsentiert wurden. Die wichtigsten Ergebnisse werden hier nochmals aufgenommen, nebeneinandergestellt und bezüglich ihrer Bedeutung für die Gleichstellung von Frauen und Männern in unserer Gesellschaft diskutiert. Insgesamt zeigen die Befunde des Freiwilligensurveys 2014, dass es hinsichtlich des freiwilligen Engagements deutliche Geschlechterunterschiede gibt. Diese Unterschiede bestehen sowohl darin, zu welchen Anteilen sich Frauen und Männer freiwillig engagieren, als auch darin, wie sie sich freiwillig engagieren. Allerdings gibt es auch viele Gemeinsamkeiten.

Unterschiede wie der Fakt, dass sich in Deutschland Frauen anteilig seltener freiwillig und öffentlich engagieren als Männer sind nicht neu, sondern aus der Engagementforschung seit Jahren bekannt (Bundesministerium für Familie, Senioren, Frauen und Jugend (BMFSFJ) 2013). International variieren Geschlechterunterschiede in der Engagementquote: Während in einigen Ländern Frauen häufiger freiwillig engagiert sind als Männer (z. B. in den USA und in Großbritannien), gibt es in anderen Ländern keine oder nur geringe Geschlechterunterschiede (z. B. Kanada) und in einer dritten Gruppe von Ländern sind
Männer häufiger freiwillig engagiert als Frauen (z.B. Deutschland und Schweden; Musick \& Wilson, 2008, S. 180). Allerdings finden sich, ähnlich wie in Deutschland, in vielen Ländern Geschlechterunterschiede in der Ausgestaltung des freiwilligen Engagements. So engagieren sich Frauen und Männer häufig in verschiedenen gesellschaftlichen Bereichen und setzen sich für verschiedene Zielgruppen ein, um zwei weitere von zahlreichen Unterschieden zwischen den Geschlechtern im Engagement zu benennen. Eine Gemeinsamkeit ist dagegen darin zu sehen, dass der Anteil Engagierter sowohl bei älteren Frauen als auch bei älteren Männern in den letzten fünfzehn Jahren deutlich gestiegen ist. Eine weitere Gemeinsamkeit liegt darin, dass sich Schülerinnen und Schüler heute zu ähnlichen Anteilen freiwillig engagieren. Was aus unserer Sicht fehlt, ist eine systematische Zusammenstellung der Unterschiede und Gemeinsamkeiten im freiwilligen Engagement von Frauen und Männern, um gegebenenfalls Implikationen zur Verbesserung der Gleichstellung von Frauen und Männern in der Zivilgesellschaft auf der Basis soliden empirischen Wissens geben zu können.

Schon im ersten Freiwilligensurvey 1999 wurde das Engagement von Frauen und Männern aus der Genderperspektive thematisiert, ebenfalls mit dem Ergebnis, dass mehr Männer als Frauen freiwillig tätig sind, dass Männer andere Tätigkeitsfelder besetzen und dass sie mehr 
Zeit für ihr freiwilliges Engagement aufwenden als Frauen (Zierau 2001). Bereits 1999 ließ sich im Engagement eine traditionelle hierarchische geschlechtsspezifische Arbeitsteilung nachzeichnen: Frauen etwa besetzen nicht nur in der Erwerbsarbeit, sondern auch im Engagement deutlich seltener Leitungs- oder Vorstandspositionen. Die Bewertung der Befunde lautete damals, dass auch im freiwilligen Engagement eine hierarchische geschlechtsspezifische Segmentierung freiwilliger Tätigkeiten vorliegt, die in Anlehnung an die Segmentierung des Arbeitsmarktes als Geschlechterungleichheit verstanden werden kann.

Nicht alle Differenzen und Unterschiede im Engagement können mit sozialer Ungleichheit der Geschlechter gleichgesetzt werden. Immer dann, wenn sich eine Geschlechterhierarchie im freiwilligen Engagement abzeichnet, die auch die geschlechtsspezifische Arbeitsteilung in anderen Lebensbereichen wie der Familie und dem Beruf widerspiegelt, ist jedoch davon auszugehen, dass mit den Geschlechterdifferenzen auch Geschlechterungleichheiten verbunden sind (Backes 2011). Wir möchten in diesem Kapitel thematisieren, ob diese Diagnose auf Basis des ersten Freiwilligensurveys 1999 auch heute noch Bestand hat, oder ob sich bezüglich der Gleichstellung von Frauen und Männern im freiwilligen Engagement in den vergangenen fünfzehn Jahren etwas verändert und gegebenenfalls verbessert beziehungsweise verschlechtert hat.

Von Gleichstellung sprechen wir in Anlehnung an den Ersten Gleichstellungsbericht der Bundesregierung, wenn gleiche Chancen für Frauen und Männer in allen Phasen des Lebensverlaufs gegeben sind, etwa bezüglich einer gleichberechtigten gesellschaftlichen Teilhabe von Frauen und Männern im Erwerbsleben, aber auch im kulturellen, wirtschaftlichen, politischen und sozialen Leben. Auch bezüglich der gesellschaftlichen Teilhabe in Form des freiwilligen Engagements sollen alle die gleichen Zugangschancen haben (Bundesministerium für Familie, Senioren, Frauen und Jugend (BMFSFJ) 2013). Der Erste Gleichstellungsbericht der Bundes- regierung betont zudem, dass insbesondere die Erweiterung von geschlechtsspezifischen Rollenbildern und damit einhergehenden Erwartungen hinsichtlich der Übernahme gesellschaftlich notwendiger bezahlter und unbezahlter Arbeit hierfür von Bedeutung sind (Bundesministerium für Familie, Senioren, Frauen und Jugend (BMFSFJ) 2013: 2). Bezüglich der Geschlechterverhältnisse im Engagement besteht nach wie vor eine Forschungslücke (Bereswill \& Braukmann 2014: 18), da häufig offen bleibt, warum Frauen und Männer welche Formen des freiwilligen Engagements unter welchen Voraussetzungen und in welchen Lebenssituationen ausüben. Dass die unterschiedlichen Rollen von Frauen und Männern in der Zivilgesellschaft in der Engagementforschung bisher vergleichsweise wenig Aufmerksamkeit erhalten haben, erscheint insbesondere vor dem Hintergrund überraschend, als dass die Gleichberechtigung von Männern und Frauen im Grundgesetz festgeschrieben ist und insbesondere den Akteuren der Zivilgesellschaft eine zentrale Funktion dabei zukommt, die Rechte von Staatsbürgerinnen und Staatsbürgern einzufordern und zu schützen (Lang \& Embacher 2015).

In diesem Kapitel möchten wir Befunde zu folgenden Forschungsfragen vorstellen und diskutieren.

1. Wie unterscheiden sich die Engagementquoten von Frauen und Männern und wie haben sich diese Unterschiede über die letzten fünfzehn Jahre verändert?

2. Welche Unterschiede und Gemeinsamkeiten zeigen sich in der Ausgestaltung des Engagements von Frauen und Männern? Gibt es eine Angleichung zwischen freiwillig engagierten Frauen und Männern?

3. Kompensiert die Beteiligung an informellen Unterstützungsleistungen die ungleiche Beteiligung im Engagement von Frauen und Männern? 
Zur Beantwortung dieser Forschungsfragen tragen wir die entsprechenden Befunde aus den empirischen Kapiteln des vorliegenden Berichts systematisch zusammen und diskutieren, inwie- weit von einer Gleichstellung der Geschlechter im freiwilligen Engagement ausgegangen werden kann und an welchen Stellen sie noch verbessert werden sollte.

\section{2 $\mathrm{Zu}$ welchen Anteilen engagieren sich Frauen und Männer?}

Als ersten Hinweis auf eine mögliche Gleichstellung im freiwilligen Engagement sind die Anteile Engagierter bei Frauen und Männern zu vergleichen. Sind die Anteile unterschiedlich hoch, so ist zu fragen, ob diese Geschlechterdifferenz auch mit einer Geschlechterungleichheit einhergeht. Sind die Anteile jedoch in etwa gleich hoch, können wir gegebenenfalls von Gleichstellung sprechen, und zwar dann, wenn das freiwillige Engagement von Frauen und Männern ähnliche Bedingungen und Gestaltungsmöglichkeiten widerspiegelt - ähnlich, da Engagement eben insbesondere durch seine Vielfalt charakterisiert werden kann. Verbergen sich jedoch hinter gleichen Anteilen Engagierter bei Frauen und Männern systematisch ungleiche Bedingungen, ist eine Gleichstellung - trotz rechtlich verankerter Gleichberechtigung - nicht gegeben. Diese Argumentation erfolgt analog der Argumentation bezüglich der Gleichstellung von Frauen und Männern in der Erwerbsarbeit. Eine Angleichung der Erwerbsquoten von Frauen und Männern führt nicht automatisch zu Gleichstellung, also etwa einer Auflösung des Lohngefälles der beiden Gruppen, da der durchschnittliche Lohnabstand zwischen Frauen und Männern (Gender Pay Gap) eben nicht allein auf unterschiedliche Erwerbsquoten oder eine unterschiedliche Zahl von Arbeitsstunden zurückzuführen ist, sondern insbesondere auch durch unterschiedliche Qualifikationsfelder und -niveaus oder auch durch die Diskriminierung von Frauen bei der Besetzung prestigeträchtiger Positionen zustande kommt.
Im Hauptbericht des Freiwilligensurveys 2014 werden deutliche Unterschiede im Engagement von Frauen und Männern aufgezeigt.

Engagementquote: Insgesamt engagieren sich Frauen mit 41,5 Prozent weiterhin zu geringeren Anteilen als Männer mit 45,7 Prozent (siehe Kapitel 3). Eine Ausnahme sind die 30- bis 49-jährigen Frauen, welche im Jahr 2014 zu einem etwas höheren Anteil engagiert sind als die Männer dieser Altersgruppe. Bei Frauen hat der Anteil der Engagierten zwischen 1999 und 2014 jedoch deutlicher zugenommen als bei Männern, sodass sich die Quoten von Frauen und Männern annähern. Eine Angleichung der Engagementquoten scheint somit in Zukunft möglich, ist aber mit Ausnahme der 30- bis 49-Jährigen noch nicht erreicht.

Die Geschlechterunterschiede in der Engagementquote finden sich auch in den meisten Bundesländern. Zwar gibt es auch einige Länder, in denen sich die Engagementquoten von Frauen und Männern nicht unterscheiden. Allerdings ist dies eher darauf zurückzuführen, dass sich Männer hier zu ebenso geringen Anteilen freiwillig engagieren wie Frauen. Im Zeitvergleich haben die Frauen zum Teil deutlich aufgeholt, zum Teil war schon in früheren Wellen kein Geschlechterunterschied in diesen Ländern festzustellen.

Niedriggebildete Frauen engagieren sich zu geringeren Anteilen als niedriggebildete Männer. Der Unterschied zwischen diesen beiden Gruppen ist im Zeitvergleich sogar größer geworden, da die Engagementquoten der niedriggebildeten Frauen weniger stark gestiegen sind als die der niedriggebildeten Männer. Niedriggebil- 
dete Frauen unterscheiden sich zudem in ihrer Engagementquote stärker von hochgebildeten Frauen als niedriggebildete Männer von hochgebildeten Männern.

Schülerinnen und Schüler engagieren sich zu gleichen Anteilen. Frauen im Alter von 14 bis 29 Jahren engagieren sich anteilig jedoch bislang etwas seltener als Männer in diesem Alter (45,0 Prozent zu 48,7 Prozent), was einen Hinweis darauf gibt, dass Barrieren für Engagement nach dem Abschluss der Schule bestehen.

Differenziert nach Erwerbsstatus zeigt sich, dass sich teilzeitbeschäftigte Frauen häufiger (52,5 Prozent) als teilzeitbeschäftigte Männer (42,7 Prozent) engagieren (siehe Kapitel 16); vollzeitbeschäftigte Frauen (42,6 Prozent) engagieren sich hingegen seltener als vollzeitbeschäftigte Männer (48,6 Prozent). Rentnerinnen engagieren sich darüber hinaus deutlich seltener als Rentner (31,0 Prozent zu 40,7 Prozent). Möglicherweise ist dies in erster Linie ein Bildungseffekt, denn in jüngeren Altersgruppen ist der Bildungsunterschied zwischen Frauen und Männern nicht mehr so ausgeprägt wie in den heute älteren Altersgruppen.

Quote öffentlicher gemeinschaftlicher Aktivität: Die Unterschiede in der Beteiligung von
Frauen und Männern sind bei den gemeinschaftlich und öffentlich ausgeübten Aktivitäten (also dem ,Mitmachen' außerhalb von Beruf und Familie) deutlich weniger ausgeprägt als im freiwilligen Engagement - nahezu gleiche Anteile von Frauen und Männern sind gemeinschaftlich aktiv (Frauen: 69,6 Prozent, Männer: 70,9 Prozent) (siehe Kapitel 3).

Früheres Engagement und Bereitschaft, sich $z u$ engagieren: Frauen haben sich zu etwas größeren Anteilen als Männer noch niemals in ihrem Leben freiwillig oder ehrenamtlich engagiert $(36,1$ Prozent zu 31,5 Prozent). Jüngere Frauen sind zu größeren Anteilen bereit, sich zukünftig zu engagieren als jüngere Männer. In der ältesten Altersgruppe ist es hingegen umgekehrt: Hier sind Frauen seltener bereit sich zu engagieren als Männer (siehe Kapitel 4).

Die Befunde sprechen im Großen und Ganzen gegen eine erreichte Gleichstellung im freiwilligen Engagement. Die Unterschiede in Bezug auf die Engagementquoten von Frauen und Männern sind insgesamt zwar nicht besonders groß, und bei den Jüngeren geringer als bei den Älteren, aber es zeigen sich einige Geschlechterunterschiede bei differenzierterer Betrachtung.

\subsection{Wie ist das Engagement von Frauen und Männern ausgestaltet?}

Hinsichtlich der Engagementquoten haben wir deutliche Unterschiede und manche Gemeinsamkeiten zwischen Männern und Frauen gefunden. Verbergen sich nun hinter ähnlich hohen Engagementquoten von Frauen und Männern sehr unterschiedliche freiwillige Tätigkeiten mit unterschiedlichem Umfang, mit unterschiedlichen Aufgaben, unterschiedlichem Einfluss und unterschiedlichem Ansehen? Und sind diese Geschlechterunterschiede im Engagement mit hierarchischen Geschlechterungleichheiten verknüpft? Dies soll wiederum in
Analogie zum Arbeitsmarkt veranschaulicht werden: Vergleicht man allein die Erwerbsquoten von Frauen und Männern, übersieht man darunterliegende Geschlechterunterschiede wie die mehrheitliche Verortung weiblicher Arbeitskräfte in prekären Arbeitsmarktsegmenten, die zu sozialer Ungleichheit in Erwerbseinkommen und Alterssicherungsansprüchen beitragen. Im Folgenden wird deshalb betrachtet, welche Unterschiede und Gemeinsamkeiten sich bezüglich freiwillig engagierter Frauen und Männer in der Ausgestaltung des Engagements zeigen. 
Zeitliche Ausgestaltung des Engagements: Es zeigen sich bedeutsame Geschlechterunterschiede hinsichtlich des Stundenumfangs und der Häufigkeit des Engagements (siehe Kapitel 12). So ist der Anteil von engagierten Frauen, die sechs Stunden pro Woche und mehr für ihre freiwillige Tätigkeit aufwenden, mit 15,0 Prozent geringer als der Anteil der engagierten Männer (21,0 Prozent). Frauen scheinen also weniger Stunden auf ihr Engagement zu verwenden als Männer. Frauen und Männer haben sich zwar zwischen 1999 und 2014 hinsichtlich des zeitlichen Umfangs der freiwilligen Tätigkeit angenähert, allerdings insbesondere weil sich Männer heute zu höheren Anteilen mit geringerem Stundenumfang engagieren als 1999. Währenddessen hat sich der Stundenumfang bei den Frauen deutlich weniger verändert. Geringe Stundenumfänge zeigen sich besonders bei Frauen im Alter von 14 bis 29 Jahren und von 30 bis 49 Jahren mit hoher Bildung. So wenden beispielsweise nur 8,3 Prozent der engagierten Frauen im Alter von 30 bis 49 Jahren mit hoher Bildung sechs und mehr Stunden pro Woche für ihr freiwilliges Engagement auf (im Schnitt aller Engagierten sind es 18,1 Prozent). Zudem üben Frauen ihre freiwilligen Tätigkeiten auch seltener aus als Männer: Lediglich ein Fünftel der Frauen, aber ein Viertel der Männer üben ihre freiwillige Tätigkeit täglich oder zumindest mehrmals in der Woche aus.

Bereiche und Zielgruppen des Engagements: Auch hinsichtlich der Zielgruppen und der Bereiche des Engagements zeigen sich bedeutsame Geschlechterunterschiede. So engagieren sich Frauen häufiger für die Zielgruppe der Kinder und Jugendlichen als Männer (siehe Kapitel 11). In den meisten Bereichen haben Frauen eine geringere und nur in wenigen Bereichen eine höhere Engagementquote als Männer (z. B. in den Bereichen Schule und Kindergarten sowie im kirchlichen oder religiösen Bereich) (siehe Kapitel 3). Hier zeigen sich Muster einer tradi- tionellen geschlechtsspezifischen Arbeitsteilung. Die Unterschiede in den Engagementbereichen zwischen Frauen und Männern zeigen sich im gesamten Lebenslauf und insbesondere im höheren Lebensalter. Auch hinsichtlich der organisationalen Struktur gibt es bedeutende Geschlechterunterschiede: So engagieren sich Frauen anteilig häufiger im organisatorischen Rahmen von Kirche und religiösen Vereinigungen sowie in individuell organisierten Gruppen und anteilig seltener im Rahmen von Vereinen und Verbänden als Männer.

Inhaltiche Ausgestaltung, Leitungsfunktionen und Geldzahlungen: Männer haben anteilig deutlich häufiger eine Leitungsfunktion im Engagement als Frauen (siehe Kapitel 11). Darüber hinaus bewerten Männer ihre Mitsprachemöglichkeiten im Engagement besser als Frauen. Weitere Unterschiede zeigen sich bei den Geldzahlungen: Männer erhalten anteilig häufiger Geld im freiwilligen Engagement als Frauen (siehe Kapitel 14). Hinsichtlich der Sachzuwendungen gibt es hingegen keine Unterschiede. Auch die Inhalte der freiwilligen Tätigkeiten, die von Männern und Frauen ausgeübt werden, sind vergleichsweise ähnlich. Eine Spezialisierung im dem Sinne, dass Frauen beispielsweise nur helfende, beratende und betreuende Tätigkeiten oder Männer nur administrative Aufgaben übernehmen, lässt sich mit unseren Befunden nicht bestätigen. Sowohl bei den Frauen als auch bei den Männern sind die Tätigkeitsinhalte des Engagements seit 1999 vielfältiger geworden.

Bezüglich der Ausgestaltung des Engagements von Frauen und Männern zeigen sich deutlich mehr Unterschiede als Gemeinsamkeiten. Frauen sind somit nicht nur zu geringeren Anteilen freiwillig engagiert, sondern wenn sie sich engagieren, dann auch zu anderen Konditionen. Von einer Gleichstellung in der Ausgestaltung des freiwilligen Engagements kann nicht gesprochen werden. 


\subsection{Kompensiert die Beteiligung in informellen Unterstützungsnetzwerken die ungleiche Beteiligung im Engagement von Frauen und Männern?}

Hinsichtlich der Frage, ob die Beteiligung an informellen Unterstützungsleistungen die ungleiche Beteiligung von Frauen und Männern im freiwilligen Engagement kompensiert, wird häufig argumentiert, dass die geringere Beteiligung von Frauen im freiwilligen Engagement auf eine höhere Beteiligung an außerfamilialen privaten informellen Unterstützungsleistungen im sozialen Nahraum zurückzuführen sei. Dies lässt sich allerdings auf Basis der Befunde des Deutschen Freiwilligensurveys 2014 nicht bestätigen. Die Beteiligung der Frauen an informellen Unterstützungsleistungen im sozialen Nahraum ist nicht überdurchschnittlich hoch ausgeprägt, somit kompensiert sie auch nicht, dass Frauen anteilig seltener freiwillig engagiert sind. Wenn auch familiale Pflege- und Betreuungsleistungen berücksichtigt werden, könnte sich das Bild möglicherweise nochmals verschieben, diese sind bei den dargestellten informellen Unterstützungsleistungen jedoch nicht berücksichtigt, weil der Fokus in unserer Studie auf den Potenzialen der zusätzlich zu familialen Leistungen möglichen gemeinschaftlichen und gesellschaftlichen Unterstützungsnetzwerke liegt.

Außerfamiliale Unterstützungsleistungen im sozialen Nahraum: Informelle Unterstützungsleistungen umfassen im Freiwilligensurvey instrumentelle Hilfeleistungen für Nachbarinnen und Nachbarn, Freundinnen und Freunde, Bekannte und Andere, die Betreuung und Pflege nicht-verwandter Personen außerhalb des eigenen Haushaltes sowie die Betreuung nicht-eigener Kinder (siehe Kapitel 9). Frauen betreuen häufiger (22,0 Prozent) Kinder, die nicht ihre eigenen sind, als Männer (15,9 Prozent). Zudem übernehmen Frauen (4,2 Prozent) häufiger die Pflege oder Betreuung von Personen im außerfamilialen sozialen Nahraum als Männer (2,6 Prozent). Männer leisten dagegen häufiger (29,7 Prozent) instrumentelle Hilfen für Personen aus der Nachbarschaft und dem Freundes- und Bekanntenkreis als Frauen (23,0 Prozent).

Freiwilliges Engagement und informelle Unterstützungsleistungen: Werden alle drei Arten informeller Unterstützungsleistungen aufsummiert, beträgt der Anteil von Personen, die Unterstützung im sozialen Nahraum leisten, 40,1 Prozent bei Frauen und 40,2 Prozent bei Männern. Frauen leisten also anteilig lediglich gleich häufig informelle Unterstützung wie Männer, insofern findet jedoch kein Ausgleich durch die informelle Unterstützung statt, der angesichts der niedrigeren Engagementbeteiligung der Frauen für gleiche gesellschaftliche Teilhabe notwendig wäre. Wie ist es nun um Geschlechterunterschiede hinsichtlich des Zusammenspiels von freiwilligem Engagement und informellen Unterstützungsleistungen bestellt? Während der Anteil von Frauen, die sich ausschließlich freiwillig engagieren, mit 21,6 Prozent etwas kleiner ist als der Anteil von Männern (24,7 Prozent), liegt der Anteil von Frauen, die keiner der beschriebenen Tätigkeiten nachgeht, mit 38,3 Prozent etwas höher als bei Männern (35,1 Prozent) (siehe Kapitel 10).

Es könnte allerdings in der Tat zutreffen, dass Frauen deshalb weniger freiwillig engagiert sind als Männer und dies auch nicht mit einem höheren Ausmaß an außerfamiliären Unterstützungsleistungen kompensieren, weil sie die Hauptlast der Familienarbeit tragen. In der Zeitverwendungserhebung 2012-2013 gibt es hinsichtlich des Umfangs der bezahlten und der unbezahlten Arbeit (hierunter fällt auch die Familienarbeit) Hinweise darauf, dass Frauen den größeren Teil der unbezahlten Arbeit übernehmen (Statistisches Bundesamt 2015). Solange Frauen jedoch mehr Familienarbeit und familiale Verpflichtungen übernehmen als Männer (Peuckert 2012), wird weder Gleichstellung in der Erwerbsarbeit noch im freiwilligen Engagement zu erreichen 
sein. Da die Aufgabenteilung in der informellen Unterstützung im sozialen Nahraum ebenfalls entlang der traditionellen geschlechtsspezifischen Arbeitsteilung orientiert ist, erzeugt diese

\subsection{Fazit}

Die Befunde des Deutschen Freiwilligensurveys 2014 sprechen im Großen und Ganzen gegen eine erreichte Gleichstellung im freiwilligen Engagement. Die Unterschiede bezüglich der Engagementquoten von Frauen und Männern sind insgesamt zwar nicht besonders groß, und bei den Jüngeren geringer als bei den Älteren, aber es zeigen sich bei differenzierterer Betrachtung doch relevante Geschlechterunterschiede. Frauen engagieren sich häufiger im Bereich Schule und Kindergarten als Männer, und sie engagieren sich häufiger für die Zielgruppe der Kinder und Jugendlichen. Diese Ergebnisse sprechen für eine nach wie vor vorhandene Orientierung an der traditionellen geschlechtsspezifischen Arbeitsteilung, die sich auch in entsprechenden Vereinbarkeitsarrangements ausdrückt: So arbeiten erwerbstätige Frauen häufig in Teilzeit, um Beruf und Familie vereinbaren zu können, gleichzeitig ermöglicht die Teilzeit auch die Vereinbarkeit dieser Verpflichtungen mit einem freiwilligen Engagement, während vollzeitbeschäftigte Frauen seltener freiwillig engagiert sind als vollzeitbeschäftigte Männer.

Zudem zeigen sich große Unterschiede innerhalb der Gruppe der Frauen. So erscheint eine Gleichstellung im freiwilligen Engagement für Frauen mit hoher Bildung in Teilen gegeben. Dies gilt aber nicht für Frauen mit niedriger Ressourcenausstattung. Im Zeitvergleich muss konstatiert werden, dass sich zwar die Situation von Frauen mit hoher Bildung im freiwilligen Engagement verbessert hat, nicht aber die Situation von Frauen mit niedriger Bildung, sodass die soziale Ungleichheit im freiwilligen Engagement sogar zugenommen hat. Insbesondere für Frauen scheinen persönliche Ressourcen wie
Art der gesellschaftlichen Arbeit kaum ausgleichende Effekte bezüglich der gesellschaftlichen Gleichstellung von Frauen und Männern insgesamt.

Bildung und Einkommen nach wie vor eine erhebliche Bedeutung im Zugang zum freiwilligen Engagement zu haben.

Trotz dieser Befundlage, die weitgehend gegen das Erreichen von Gleichstellung im freiwilligen Engagement spricht, gibt es auch Befunde, die für eine zumindest künftige Verbesserung $\mathrm{zu}$ sprechen scheinen. Eine zukünftige Gleichstellung erscheint möglich, aber sie ist an Voraussetzungen gebunden. Die gleiche Beteiligung von Frauen und Männern ist nur dann möglich, wenn auch in anderen Lebensbereichen eine vollständige Arbeitsteilung zwischen Frauen und Männern erreicht wird. Solange Frauen die Hauptlast der Familienarbeit zu tragen haben, wird also sehr wahrscheinlich auch keine Gleichstellung im freiwilligen Engagement zu erreichen sein. Diese Argumentation kann belegt werden durch die Hinderungsgründe, die von noch nie engagierten Personen im Freiwilligensurvey genannt werden: 47,2 Prozent der noch nie engagierten Frauen, aber 56,1 Prozent der noch nie engagierten Männer nennen berufliche Gründe als Hinderungsgründe. Mit 38,7 Prozent sind familiäre Gründe bei Frauen fast genauso wichtig wie berufliche, bei Männern hingegen wird dies mit 24,1 Prozent deutlich seltener genannt.

Im Gleichstellungsbericht der Bundesregierung werden verschiedene Formen der gesellschaftlich notwendigen Arbeit betrachtet, hierzu zählen die Erwerbsarbeit, die unbezahlte Hausarbeit, Hilfeleistungen für Verwandte und Bekannte sowie gemeinnützige und ehrenamtliche Tätigkeiten (Bundesministerium für Familie, Senioren, Frauen und Jugend (BMFSFJ) 2013: 237). Als Hauptergebnis bezüglich des freiwilligen Engagements wird dort festgehalten: 
Frauen engagieren sich weniger als Männer in ehrenamtlichen Tätigkeiten, haben weniger ehrenamtliche Leitungsfunktionen inne und richten ihr Engagement stärker an der Familienphase orientiert und im sozialen Bereich aus, da sie es mit ihrer hohen Zeitbindung durch Haus- und Sorgearbeit verknüpfen (Bundesministerium für Familie, Senioren, Frauen und Jugend (BMFSFJ) 2013: 238).

Dieser Befund lässt sich im Kern auch durch den Deutschen Freiwilligensurvey 2014 stützen. In Deutschland wird zwar viel für eine verbesserte Vereinbarkeit von Familie und Beruf getan, eine faktische und vollständige Gleichstellung von Frauen und Männern ist jedoch im freiwilligen Engagement derzeit ebenso wenig erreicht wie auf dem Arbeitsmarkt. Die überwiegende Mehrheit der empirischen Ergebnisse spricht dagegen, und zwar trotz der Annäherung der Engagementquoten von Frauen und Männern über einen Zeitraum von fünfzehn Jahren.

Allerdings sind häufig nur graduelle Unterschiede zwischen den Geschlechtern zu beschreiben, während die Unterschiede etwa zwischen den Bildungsgruppen wesentlich größer erscheinen (siehe Kapitel 25). Zudem haben sich für manche Gruppen von Frauen durchaus Veränderungen in Hinsicht auf die Möglichkeit von Gleichstellung im Engagement ergeben, zum Beispiel bei den Schülerinnen und Schülern sowie den Frauen im Alter von 30 bis 49 Jahren und den Frauen mit hoher Bildung. Es besteht also eine Heterogenität innerhalb der Gruppe der Frauen, die bei entsprechenden Maßnahmen zur Verbesserung der Rahmenbedingungen für freiwilliges Engagement ebenfalls Berücksichtigung finden sollte. Fraglich bleibt allerdings, ob eine Zunahme des Engagements von Frauen (etwa im Bereich Schule und Kindergarten), wirklich $\mathrm{zu}$ mehr Gleichstellung beiträgt, oder ob nicht Geschlechterungleichheiten aus anderen Lebensbereichen dadurch faktisch zementiert werden (vgl. Alscher 2010).

Die Aufnahme einer freiwilligen Tätigkeit ist abhängig von Motiven, verfügbaren Ressourcen wie Geld, Bildung und Zeit sowie der Einbindung in Netzwerke, die mehr oder weniger mobilisierend wirken, so argumentieren Verba et al. mit ihrem ,Civic Voluntarism Model' (Verba, Schlozman \& Brady 1995). Frauen und Männer können sich in verschiedenen Lebensphasen bezüglich ihrer Motive, Ressourcen und Netzwerke deutlich unterscheiden. Die gesellschaftliche Arbeitsteilung kann als eine zentrale Rahmenbedingung der Unterschiede im Engagement betrachtet werden. Des Weiteren werden Geschlechterunterschiede im Engagement in der Literatur zurückgeführt auf eine unterschiedliche Sozialisation. Zudem verfügen Frauen über Netzwerke, die möglicherweise weniger engagementförderlich sind. Frauen haben darüber hinaus mehr Zeitrestriktionen aufgrund der Vereinbarkeit von Familie und Beruf; möglicherweise werden sie bei der Vergabe von begehrten freiwilligen Positionen aber auch benachteiligt (Kletzing \& Lukoschat 2014).

Es wird davon ausgegangen, dass nicht nur individuelles Verhalten von Frauen und Männern Geschlechterunterschiede (re-)produziert, sondern auch die jeweiligen strukturellen Rahmenbedingungen zur Aufrechterhaltung der Geschlechterhierarchien beitragen können (Gottschall 2000: 296ff.). Sozialpsychologische Ansätze gehen davon aus, dass sich Geschlechtsrollenstereotype auf Verhalten im Allgemeinen und auf soziale Interaktionen im Speziellen auswirken (Green, Ashmore \& Manzi Jr 2005; Hannover 2010). Somit können Geschlechtsrollenstereotype beispielsweise auch eine Rolle bei der Entscheidung für oder gegen bestimmte Engagementbereiche spielen.

Das Geschlecht einer Person ist insofern von Bedeutung, als dass es als eine Strukturkategorie aufgefasst werden kann, die verschiedene Bereiche der Gesellschaft strukturiert und die häufig mit einem Macht- und Hierarchieverhältnis der Geschlechter verknüpft ist. Dies betrifft nicht nur die Arbeitsteilung einer Gesellschaft, sondern auch den Zugang zu politischen, ökonomischen und kulturellen Ressourcen (Bereswill 2008: 102). Darüber hinaus hat sich in der soziologi- 
schen Debatte zur Analyse sozialer Ungleichheit ein Konzept der Intersektionalität etabliert, das die Wechselwirkungen zwischen Geschlecht und anderen Ungleichheitskategorien wie etwa Klasse und Ethnizität für die (Re-)Produktion sozialer Ungleichheit hervorhebt, die über eine bloße Addition der Kategorienzugehörigkeit hinausreicht (Winker \& Degele 2009). Im freiwilligen Engagement wird dessen Erkenntnisgewinn beispielsweise bezüglich der geringen Engagementbeteiligung von niedriggebildeten Frauen deutlich.

Als Ziel formuliert der Erste Gleichstellungsbericht:

Ziel ist es, beiden Geschlechtern zu ermöglichen, neben der Erwerbsarbeit auch andere gesellschaftlich notwendige und wertvolle Formen von Arbeit (Hausarbeit, Sorgearbeit, freiwilliges Engagement, Nachbarschaftshilfe) in ihren Alltag und entlang ihres Lebenslaufs zu integrieren. Dieses Erfordernis betrifft vor allem die Familiengründungsphase, aber auch die mittlere Lebensphase, in der Betreuungs- und Pflegeleistungen für bedürftige ältere Familienangehörige, Verwandte und Freunde anfallen - voraussichtlich mit steigender Tendenz. (Bundesministerium für Familie, Senioren, Frauen und Jugend (BMFSFJ) 2013: 244)

Um diesem Ziel der Gleichstellung von Frauen und Männern näher zu kommen, können verschiedene Akteure im Feld des freiwilligen Engagements ihren Beitrag leisten, nicht zuletzt die Frauen und Männer selbst. Darüber hinaus sollten engagementpolitische Akteure Fragen der Gleichstellung von Frauen und Männern stärker in den Blick nehmen, und zwar auf der Bundesebene, der Landesebene sowie auch auf der Ebene der Kommunen. Ebenso sind aber auch die Organisationen der Zivilgesellschaft gefordert ihren Beitrag zur Gleichstellung von Frauen und Männern zu leisten. All diesen Akteuren kommt eine wichtige Rolle $\mathrm{zu}$, um Lösungen und Arrangements für eine gesamtgesellschaftliche Arbeitsteilung zu finden, in der Frauen und Männer tatsächlich gleichgestellt sind. Im letzten Kapitel des vorliegenden Berichts gehen wir auf entsprechende Implikationen im Bereich des freiwilligen Engagements nochmals näher ein.

\section{Literatur}

Alscher, M. (2010). Auf der Suche nach Balance. Frauen und Männer zwischen Beruf, Familie und Engagement. WZB Mitteilungen, 2010(129), 31-33.

Backes, G. M. (2011). Geschlechterdifferenz im Engagement. In: T. Olk \& B. Hartnuß (Hrsg.) Handbuch Bürgerschaftliches Engagement (S. 65-75). Weinheim: Beltz Juventa.

Bereswill, M. (2008). Geschlecht. In: N. Baur, H. Korte, M. Löw \& M. Schroer (Hrsg.) Handbuch Soziologie (S. 97-116). Wiesbaden: VS Verlag für Sozialwissenschaften.

Bereswill, M., \& Braukmann, S. (2014). Fürsorge und Geschlecht. Neue und alte Geschlechterkonstellationen im freiwilligen Engagement Älterer. Weinheim: Beltz Juventa.

Bundesministerium für Familie, Senioren, Frauen und Jugend (BMFSFJ) (2013). Neue Wege - Gleiche Chancen. Gleichstellung von Frauen und Männern im Lebensverlauf. Erster Gleichstellungsbericht. Berlin: BMFSFJ.

Gottschall, K. (2000). Soziale Ungleichheit und Geschlecht. Opladen: Leske + Budrich.

Green, R. J., Ashmore, R. D., \& Manzi Jr, R. (2005). The structure of gender type perception: Testing the elaboration, encapsulation, and evaluation framework. Social Cognition, 23(5), 429-464.

Hannover, B. (2010). Sozialpsychologie und Geschlecht: Die Entstehung von Geschlechtsunterschieden aus der Sicht der Selbstpsychologie. In: G. Steins (Hrsg.) Handbuch Psychologie und Geschlechterforschung (S. 27-42). Wiesbaden: VS Verlag für Sozialwissenschaften. 
Kletzing, U., \& Lukoschat, H. (2014). Engagiert vor Ort - Wege und Erfahrungen von Kommunalpolitikerinnen. Berlin: Bundesministerium für Familie, Senioren, Frauen und Jugend.

Lang, S., \& Embacher, S. (2015). Engagementpolitik nach Michael Bürsch - Mehr Demokratie wagen. In: S. Embacher \& S. Lang (Hrsg.) Recht auf Engagement. Plädoyers für die Bürgergesellschaft. Bonn: Dietz.

Musick, M. A., \& Wilson, J. (2008). Volunteering: A social profile. Bloomington: Indiana University Press.

Peuckert, R. (2012). Familienformen im sozialen Wandel. Wiesbaden: Springer VS.

Statistisches Bundesamt (2015). Wie die Zeit vergeht. Ergebnisse zur Zeitverwendung in Deutschland 2012/2013. Wiesbaden: Statistisches Bundesamt.

Verba, S., Schlozman, K. L., \& Brady, H. E. (1995). Voice and equality. Civic voluntarism in American politics. Cambridge: Harvard University Press.

Winker, G., \& Degele, N. (2009). Intersektionalität. Bielefeld: transcript.

Zierau, J. (2001). Genderperspektive - Freiwilligenarbeit, ehrenamtliche Tätigkeit und bürgerschaftliches Engagement bei Männern und Frauen In: S. Picot (Hrsg.) Freiwilliges Engagement in Deutschland. Freiwilligensurvey 1999. Ergebnisse der Repräsentativerhebung zu Ehrenamt, Freiwilligenarbeit und bürgerschaftlichem Engagement. Band 3: Frauen und Männer, Jugend, Senioren, Sport (S. 15-110). Stuttgart: Kohlhammer.

Open Access Dieses Kapitel wird unter der Creative Commons Namensnennung 4.0 International Lizenz (http://creativecommons.org/licenses/by/4.0/deed.de) veröffentlicht, welche die Nutzung, Vervielfältigung, Bearbeitung, Verbreitung und Wiedergabe in jeglichem Medium und Format erlaubt, sofern Sie den/die ursprünglichen Autor(en) und die Quelle ordnungsgemäß nennen, einen Link zur Creative Commons Lizenz beifügen und angeben, ob Änderungen vorgenommen wurden.

Die in diesem Kapitel enthaltenen Bilder und sonstiges Drittmaterial unterliegen ebenfalls der genannten Creative Commons Lizenz, sofern sich aus der Abbildungslegende nichts anderes ergibt. Sofern das betreffende Material nicht unter der genannten Creative Commons Lizenz steht und die betreffende Handlung nicht nach gesetzlichen Vorschriften erlaubt ist, ist für die oben aufgeführten Weiterverwendungen des Materials die Einwilligung des jeweiligen Rechteinhabers einzuholen. 